CDF/EXOTIC/PHYS/CDFR/6185

Version 3.00

December 4, 2002

\title{
CDF Tau Triggers, Analysis, and Other Developments
}

\author{
John R. Smith \\ University of California at Davis \\ (CDF Collaboration)
}

Summary. This note is a write-up of contribution made by the author to the HCP2002 conference. It has two principal subjects.

The first subject concerns the CDF $\tau$ triggers, $\tau$-cone algorithms and $\tau$ physics analysis. $\tau$ physics is going to be very important in Run II because $\tau$ 's can extend SUSY searches at large $\tan \beta$ in particular, $\tau$ 's will help in the searches for $\tilde{\chi}_{1}^{ \pm} \tilde{\chi}_{2}^{0}$, MSSM Higgs and other non Standard Model (SM) processes. Also, $\tau$ events are important for various Standard Model processes including Precision Electroweak, $t \bar{t}$, and SM Higgs searches. $\tau$ triggers are installed and operating at CDF.

The second subject of this contribution to the HCP2002 conference concerns the algorithms of backwards differentiation abstracted from their usual setting inside of Automatic Differentiation software packages. Backwards differentiation (reversemode differentiation) provides a useful means for optimizing many kinds of problems. 


\section{Introduction}

There are many physics advantages for setting up detector triggers that collect events containing $\tau$ 's. In particular, there is a great deal of Standard Model (SM) physics which involves $\tau$ production or "pencil-like" jets at moderate $p_{T}$. For example, production of charm or B states such as $\Upsilon$, $\left(B^{ \pm}, B^{0}, B_{s}\right)$ mesons or $\left(D^{ \pm}, D^{0}, D_{s}\right)$ mesons all involve final states which can contain $\tau$ mesons or "pencil-like" jets, since low transverse energy $\left(E_{T}\right)$ $b \bar{b}$ or $c \bar{c}$ jets also have "pencil-like" characteristics.

SM physics at high transverse momentum $\left(p_{T}\right)$ involving $\tau$ 's include DrellYan production of $\tau^{+} \tau^{-}$pairs, $t \bar{t}$ channel with $W \rightarrow \tau \nu_{\tau}$ and associated Higgs production $\left(W H^{0}\right.$ or $Z H^{0}$ ) with $W / Z \rightarrow \tau$ or jet and $H^{0} \rightarrow b \bar{b} / \tau^{+} \tau^{-}$.

Also, $\tau$ 's are important for studying physics beyond the SM, e.g., Supersymmetry (SUSY) searches such as $\mathrm{p} \overline{\mathrm{p}} \rightarrow \tilde{\chi}_{1}^{ \pm} \tilde{\chi}_{2}^{0}, \mathbb{R}_{p}$ SUSY scenarios, MSSM Higgs, etc. As shown in Fig. 1, the leptonic branching ratios of $\tilde{\chi}_{1}^{ \pm} \rightarrow l^{ \pm} \nu \tilde{\chi}_{1}^{0}$

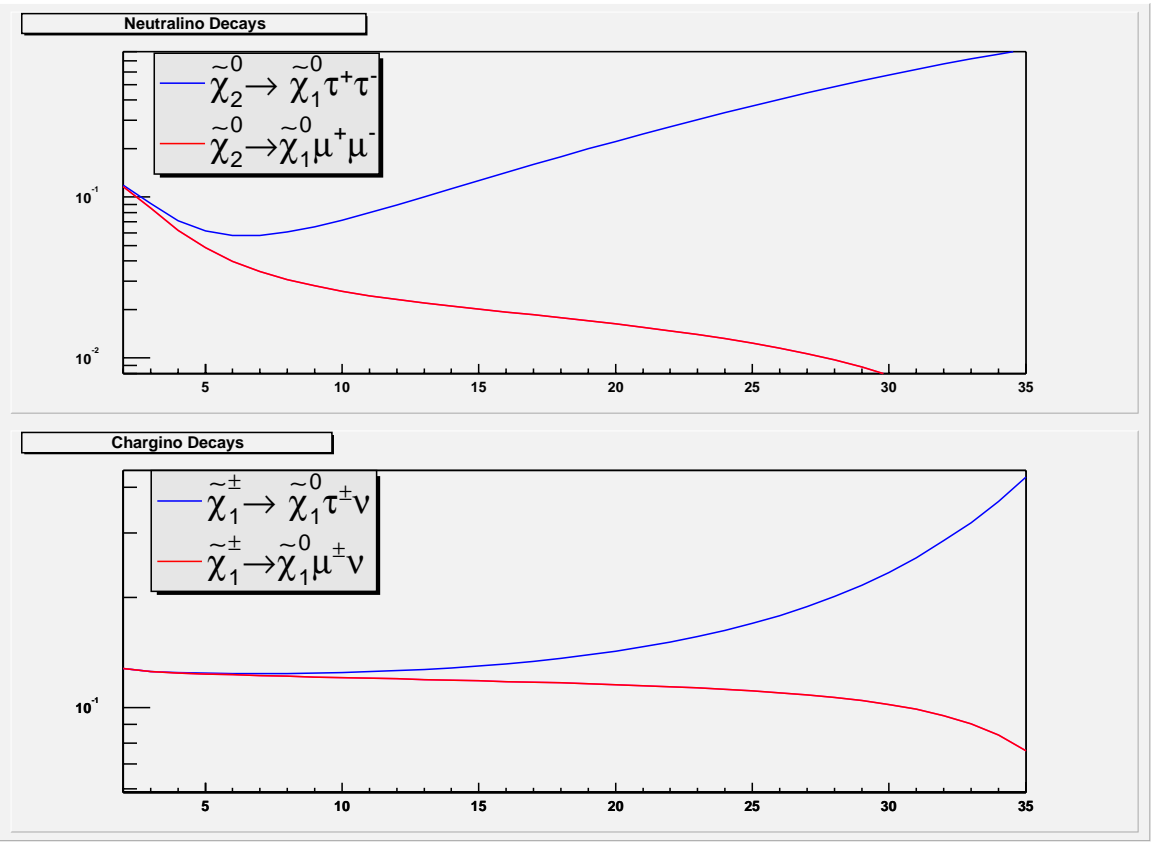

Fig. 1. Branching ratio for chargino and neutralino decays as a function of $\tan \beta$. ISAJET 7.63, (mSUGRA): $m_{0}=150, m_{1 / 2}=150, A_{0}=0, \operatorname{sign}(\mu)=+1$

and $\tilde{\chi}_{2}^{0} \rightarrow l^{+} l^{-} \tilde{\chi}_{1}^{0}$ are strongly dependent on the SUSY parameter, $\tan \beta$. Therefore, including $\tau$ events is important in extending the reach of SUSY searches to the high $\tan \beta$ region of parameter space. The previous CDF Run 
I searches for SUSY in the tri-lepton channels was based on four channels: $e e e, e e \mu, e \mu \mu$ and $\mu \mu \mu$ because there was no way to trigger on $\tau$ events for that data sample. Depending on the $\int \mathcal{L} d t$, inclusion of $\tau$ 's can substantially extend the reach of SUSY searches.

\section{Run II Trigger System and Tau Triggers}

The CDF detector and DAQ have been extensively upgraded for Run II, see [1] which contains a description of the detector components.

Fig. 2 illustrates the new CDF "Deadtimeless" data flow and the 3-Level Trigger System. To achieve the "Deadtimeless" operation the data flow is pipelined at Level 1 and buffered at Level 2. At Level 3 the events are analyzed with full even reconstruction using a processor farm. The CDF $\tau$ triggers are integrated into all 3 levels of the trigger system. CDF has 5 different $\tau$ triggers operating presently: they are (1) Central Muon plus track, (2) Central Muon Extension plus track, (3) Electon plus track, (4) Di-Tau and (5) Tau $+\mathbb{F}_{T}$ triggers. In Trigger (5) $\mathbb{F}_{T}$ is determined by taking the negative of the vector sum of the calorimeter cells with energy above a threshold of $1 \mathrm{GeV}$ in the transverse plane. Triggers (1) through (3) are designed for leptonic tau decays, whereas triggers (4) and (5) are designed for hadronic tau decays. The heart of these triggers is an algorithm defining a "tau-cone object." The essential part of this algorithm is schematically shown in Fig. 3. To initialize the construction of the tau-cone object, a seed direction is determined either from calorimeter clusters or tracks (above suitable thresholds in $E_{T}$ or $p_{T}$ respectively). Then the region within a $10^{\circ}$ angle from the seed direction is used to define the cone of tracks to be associated with the $\tau$. The region between $10^{\circ}$ and $30^{\circ}$ defines the cone of isolation. We expect to see no tracks in the cone of isolation associated with the charged decays of a $\tau$. Therefore, the tau-algorithm demands that there be zero tracks found in the cone of isolation, $N_{\text {track }}^{10^{\circ}-30^{\circ}}=0$.

Table 1 contains detailed description of the Electron plus track trigger (see [1] for a description of the detector components). The following definitions apply: $E M$ is electromagnetic shower energy; XFT is the extremely fast track processor; Had is hadronic energy; XCES is the Central Shower Maximum finder; $\phi$ is the azimuthal angle; $\Delta Z_{C E S}$ is the z-separation of the extrapolated track position at the XCES position; $\chi_{C E S}^{2}$ measures deviations between the XCES profile and reference profiles; $\eta=\log (\tan (\theta / 2))$, where $\theta$ is the spherical polar coordinate; $\Delta Z_{0}$ is the difference in $\mathrm{z}$ positions at the distance of closest approach to the beam; and $\Delta R=\sqrt{(\Delta \eta)^{2}+(\Delta \phi)^{2}}$.

\section{Finding Taus: $W \rightarrow \tau \nu$ first result}

The above triggers have already played a role in find taus in the Run II data. One of the large cross section Electroweak processes is $p \bar{p} \rightarrow W \rightarrow \tau \nu$. Events 
Table 1. Electron + track trigger

\begin{tabular}{|c|c|}
\hline Level 1 & $\begin{array}{l}\text { EM Shower with XFT track } \\
\qquad E_{T}(e)>8 \mathrm{GeV}, \mathrm{Had} / E M<1 / 8 \\
\text { Associated XFT } p_{T} \geq 8.34 \mathrm{GeV} / \mathrm{c}\end{array}$ \\
\hline Level 2 & $\begin{array}{l}\text { EM Shower Cluster } \\
\qquad E_{T}(e)>8 \mathrm{GeV}, \mathrm{XCES}>2 \mathrm{GeV} \\
H a d / E M<1 / 8 \\
\text { 2nd XFT track } \\
\quad p_{T}>5.18 \mathrm{GeV} / \mathrm{c} \\
\mid \Delta \phi(e, \text { track }) \mid>10^{\circ}\end{array}$ \\
\hline Level 3 & $\begin{array}{l}\text { electron matched to EM Shower } \\
\qquad\left|\Delta z_{\mathrm{CES}}\right|<8 \mathrm{~cm}, \chi_{\mathrm{CES}}^{2}<20 \\
E_{T}>8 \mathrm{GeV}, p_{T}>8 \mathrm{GeV} / \mathrm{c} \\
\tau \text {-cone track requirements } \\
\quad p_{T} \geq 5 \mathrm{GeV} / \mathrm{c},|\eta| \leq 1.5 \\
N_{\text {track }}^{10^{\circ}-30^{\circ}}=0 \\
\quad\left(p_{T}>1.0 \mathrm{GeV} / \mathrm{c} \text { and }|\Delta z|<15 \mathrm{~cm}\right) \\
\text { electron }+\tau \text {-cone track object } \\
\quad\left|\Delta Z_{0}\right|<15 \mathrm{~cm},|\Delta R|>0.175\end{array}$ \\
\hline
\end{tabular}

were selected with a "mono-jet" plus $\mathbb{F}_{T}$ topology using the cuts shown in Table 2 .

The charge multiplicity associated with the $\tau$ candidate found in events passing the above cuts is shown in Fig. 4 for $15 \mathrm{pb}^{-1}$ of data. The data points are compared with various Monte Carlo (MC) calculations including the signal MC ( $W \rightarrow \tau \nu)$ as well as various backgrounds which pass the analysis cuts. For $\tau$ decays, one expects to see an odd charged multiplicity. Fig. 4 was made without any requirements on the charged tracks other than the above mentioned tau-cone requirements.

\section{Fast Derivatives: Backwards Differentiation}

I summarize here a useful set of algorithms which find application in many areas of research[2]. The basic problem under consideration here is how to find an efficient method of computing derivatives of a function of many parameters, i.e., given $f\left(x_{1}, x_{2}, \ldots, x_{n}\right)$ what is the fastest method to obtain $\nabla f$, or $\partial^{2} f / \partial x_{i} \partial x_{j}$ ?

There are techniques used in Automatic Differentiation packages based on reverse-mode differentiation which can be used to solve the above problem 


\section{Dataflow of CDF "Deadtimeless" \\ Trigger and DAQ}

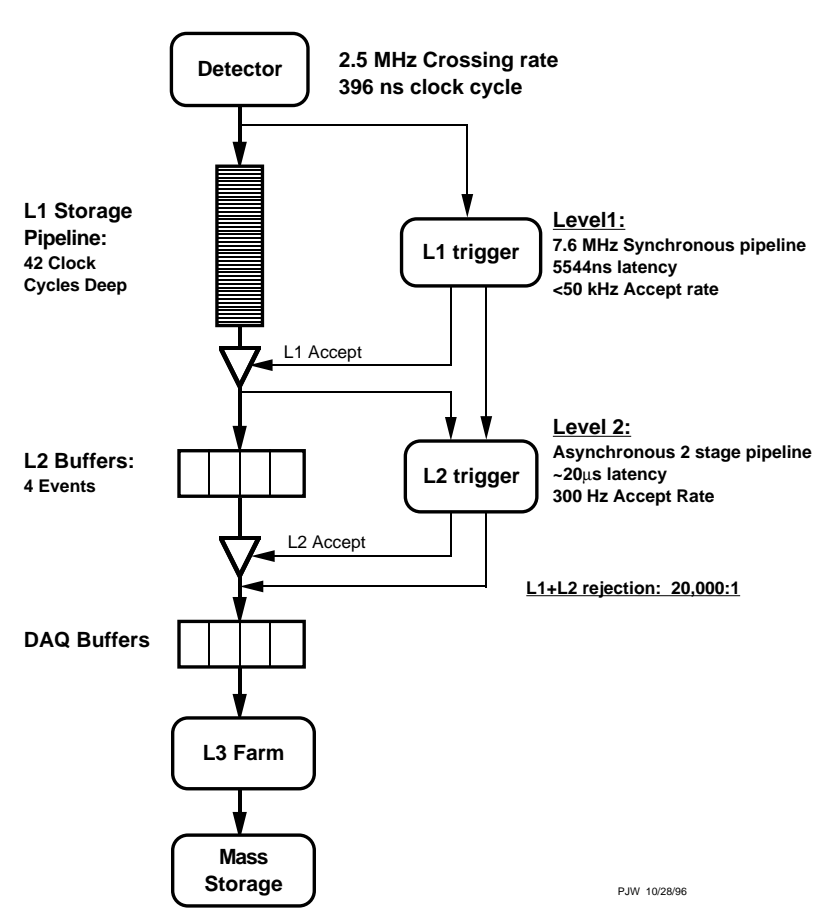

Fig. 2. Limits: L1-40 kHz, L2-300 Hz, L3-40 Hz

L1 and L2 Custom Designed VME crates

L3 Processor Farms with event reconstruction

efficiently. Such algorithms are usually incorporated into "black box" programs, and find applications in many areas such as Gradient Descent problems, Kalman Filtering (tracking), alignment problems, Back-propagation in Artificial Neural Nets (ANN), multivariate $\chi^{2}$ minimization via the NewtonRaphson method, maximum likelihood estimation, finite element analysis, etc. It would be useful to abstract the essence of reverse-mode differentiation out of the "black box" so that the basic method can be seen and understood by anyone using these procedures.

Let us begin by considering an ordered list of computations for the function $f\left(x_{1}, x_{2}, \ldots, x_{n}\right)$, 


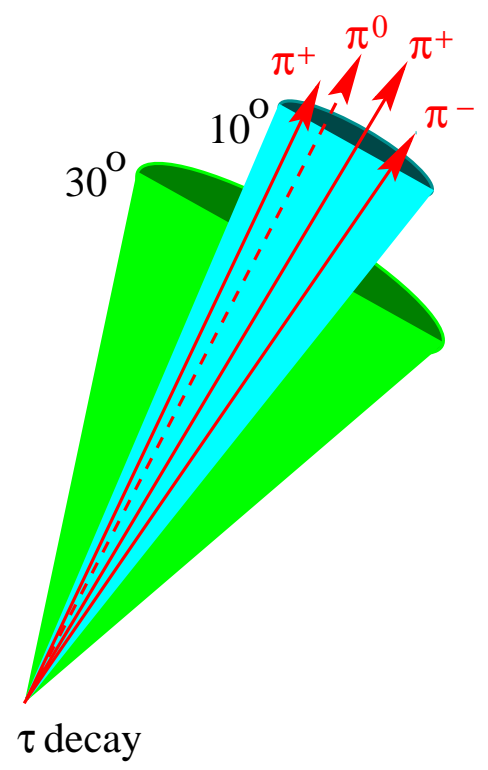

Fig. 3. Cones for $\tau$-trigger and Analysis.

Table 2. Event Selection for $W \rightarrow \tau \nu$ Analysis

\begin{tabular}{|c|c|}
\hline One central hadronic $\tau$ candidate & 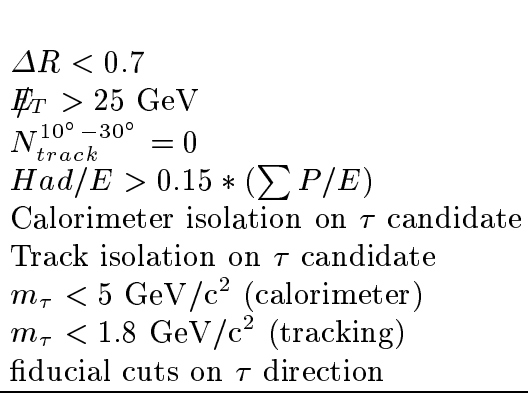 \\
\hline $\mathscr{H}_{T}>25 \mathrm{GeV}$ (calculated from $\tau$ seed) & \\
\hline no other jets in event with $E_{T}>5 \mathrm{GeV}$ & \\
\hline no interacting cosmic ray events & \\
\hline
\end{tabular}




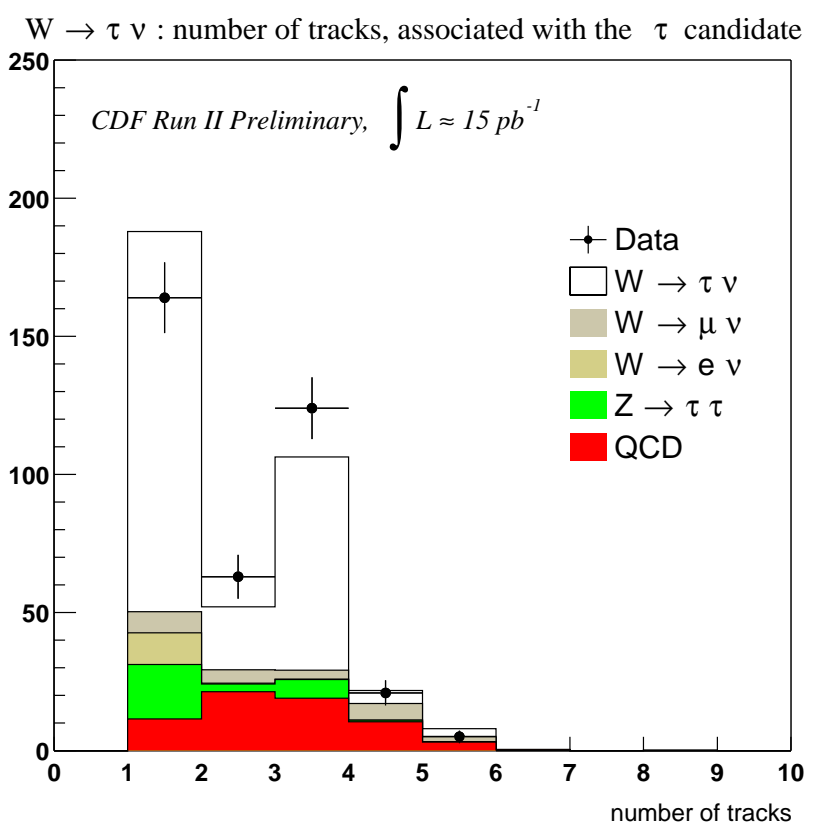

Fig. 4. Charged Multiplicity associated with $\tau$ candidate.

$$
\begin{aligned}
h_{1} & =f_{1}\left(\Omega_{1}\right) \\
h_{2} & =f_{2}\left(\Omega_{2}\right) \\
h_{3} & =f_{3}\left(\Omega_{2}\right) \\
& \vdots \\
h_{r} & =f_{r}\left(\Omega_{r}\right)
\end{aligned}
$$

where $f\left(x_{1}, x_{2}, \ldots, x_{n}\right)=h_{r}, \Omega_{k} \subseteq \boldsymbol{x} \cup\left\{h_{i}: i<k\right\}$ and $\boldsymbol{x}$ is a set of $n$ parameters. To simplify the notation that follows, assign the set of parameters to the ordered list $h_{1}=x_{1}, h_{2}=x_{2}, \ldots, h_{n}=x_{n}$, so we may assume $\Omega_{k} \subseteq$ $\left\{h_{i}: i<k\right\}$. It is important to remember that $h_{r}$ represents our computation of $f\left(x_{1}, x_{2}, \ldots, x_{n}\right)$ (the resulting computation of $n$-lines of assignments from a computer program).

The straight-forward approach, called Forward Differentiation, is based on the chain-rule of Calculus, i.e., beginning with the first line and proceeding forward, starting with $i=1$,

$$
\frac{\partial h_{i}}{\partial x_{j}}=\sum_{k=1}^{i-1} \frac{\partial f_{i}}{\partial h_{k}} \frac{\partial h_{k}}{\partial x_{j}}
$$

and continuing until $\mathrm{i}=\mathrm{r}$. Computation of $\nabla f$ takes one-pass through entire list for each parameter $x_{j}$. This method require $n$ passes for $n$ parameters 
and is slow for large $n$. The object is to find an improved method to compute $\nabla f$ that can take advantage of overwriting to limit space requirements.

In that spirit, here are the Rules for Backward Differentiation applied to the calculation of $\nabla f$. Let $\boldsymbol{F}$ be an array of length $r$. Start at the bottom of

the List of Computations and work backward towards the beginning of the list.

- Initialize: $F_{i}=0$, for $i<r, F_{r}=1$

- $F_{i} \rightarrow F_{i}+F_{k}\left[\partial f_{k} / \partial h_{i}\right]$, for all $i$ where $h_{i} \in \Omega_{k}, k=r, r-1, \ldots, n+1$

- $\nabla f: \partial f / \partial x_{i}=F_{i}, i=1,2, \ldots, n$

- and, in general, $\partial f / \partial h_{i}=F_{i}, i=1,2, \ldots, r$

- $\nabla f$ computed in one backwards pass!

One must be able to determine $i$ and $k$ going backward and compute $\partial f_{k} / \partial h_{i}$ dynamically from $h_{1}, h_{2}, \ldots, h_{r}$.

As a second example, the Hessian: $\partial^{2} f / \partial x_{v} \partial x_{i}$, can be computed by applying the backward differentiation algorithm twice. Let $\boldsymbol{F}$ be provided from before and let $\boldsymbol{S}$ and $\boldsymbol{Q}$ be arrays of length $r$.

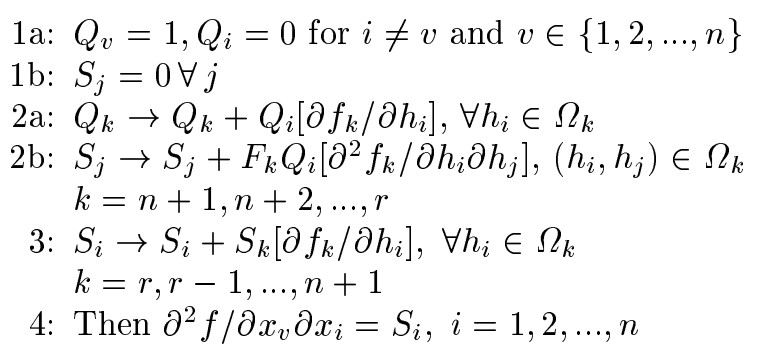

N.B. $\boldsymbol{Q}$ calculated by forward differentiation (Step-3 $\rightarrow$ first derivative calculation). The above algorithm computes one row of $\partial^{2} f / \partial x_{v} \partial x_{i}$ for each $v$. The $n \times n$ Hessian matrix of second derivatives is computed by the above technique with only $n$ passes and space requirements are kept to a manageable length by overwriting.

\section{References}

1. The CDF II Detector: Technical Design Report, November 1996, Fermilab-Pub96/390-E.

2. A. Griewank, F. Corliss, Automatic Differentiation of algorithms: Theory, Implementation and Application, SIAM, Philadelphia, 1991; Computational Differentiation: Techniques, Applications, and Tools M. Berz, C. Bischof, A. Griewank, G. Corliss (Eds.), (SIAM, Philadelphia, 1996), ISBN 0-89871-385-4. 